\title{
Multiple-Symbol Decision-Feedback Space-Time Differential Decoding in Fading Channels
}

\author{
Yan Liu \\ Schlumberger Austin Technology Center, Austin, TX, USA \\ Email:yanliu@ee.tamu.edu \\ Xiaodong Wang \\ Department of Electrical Engineering, Columbia University, New York, NY 10027, USA \\ Email:wangx@ee.columbia.edu
}

Received 20 May 2001 and in revised form 5 January 2002

\begin{abstract}
Space-time differential coding (STDC) is an effective technique for exploiting transmitter diversity while it does not require the channel state information at the receiver. However, like conventional differential modulation schemes, it exhibits an error floor in fading channels. In this paper, we develop an STDC decoding technique based on multiple-symbol detection and decisionfeedback, which makes use of the second-order statistic of the fading processes and has a very low computational complexity. This decoding method can significantly lower the error floor of the conventional STDC decoding algorithm, especially in fast fading channels. The application of the proposed multiple-symbol decision-feedback STDC decoding technique in orthogonal frequency-division multiplexing (OFDM) system is also discussed.
\end{abstract}

Keywords and phrases: space-time differential block code (STDC), flat-fading, multiple-symbol detection, decision-feedback, OFDM.

\section{INTRODUCTION}

Space-time coding (STC) methodologies, which integrate the techniques of antenna array spatial diversity and channel coding, can provide significant capacity gains in wireless channels. There have been many recent works addressing the design and applications of STC, for example, $[1,2,3,4,5]$. Meanwhile, research on receiver design for STC systems is also active [6]. Thus far, most research in this area has assumed that the fading channel state information (CSI) is available at the receiver for coherent detection. When no CSI is available, the transmission of training symbols is then necessary. This is reasonable when the channel changes slowly compared with the symbol rate, since the transmitter can send training symbols which enable the receiver to estimate the channel. However, channel estimation introduces additional complexity cost. In addition, if the channel experiences fast fading, channel estimation becomes more difficult and may require too many training symbols. Therefore under these situations, it is better to avoid channel estimation. Recently, space-time differential coding (STDC) has been developed in $[7,8]$. However, in these works the channel is assumed to be quasi-static. When employed in fading channels, the simple differential space-time decoding method exhibits an error floor, just like the conventional differential demodulation schemes. In this paper, we develop a multiplesymbol decision-feedback decoding technique for decoding STDC in fading channels, which considerably reduces the error floor, especially in fast fading channels.

For systems with single transmit antenna, it is known that multiple-symbol differential detection $[9,10,11,12]$ can eliminate the error floor in fading channels at the expense of increased receiver complexity. In [13], a multiplesymbol decision-feedback differential detection scheme was proposed, which has a very low complexity. Although this scheme cannot eliminate the error floor of the simple differential detector, it can significantly reduce such a floor. The multiple-symbol decision-feedback STDC decoding algorithm developed in this paper is a generalization of this technique to systems employing multiple transmit antennas and space-time differential coding.

Orthogonal frequency-division multiplexing (OFDM) is a multi-carrier digital modulation technique whose popularity is rising. In OFDM $[14,15]$, the entire channel is divided into many narrow sub-channels through which data are transmitted in parallel, thereby increasing the symbol duration and reducing intersymbol interference. OFDM transforms a frequency-selective fading channel into a set of parallel flat-fading channels. Recent works $[16,17,18]$ have addressed channel estimation in OFDM systems employing 
multiple antennas and space-time coding. In this paper, we will address the application of STDC in OFDM systems and the corresponding multiple-symbol decision-feedback differential receiver.

The rest of this paper is organized as follows. In Section 2, we summarize the space-time differential code (STDC) and its decoding algorithm in additive white Gaussian noise (AWGN) channels. In Section 3, we derive the decisionfeedback multiple-symbol STDC decoding algorithm. In Section 4, we discuss the application of STDC in OFDM systems and the corresponding multiple-symbol decisionfeedback differential receiver. Section 5 contains the conclusions.

\section{SPACE-TIME DIFFERENTIAL BLOCK CODING}

Space-time differential block coding was developed in $[7,8]$. Consider a communication system with two transmit antennas and one receive antenna. Let the MPSK information symbols at time $n$ be

$$
a_{n} \in \mathscr{A} \triangleq\left\{\frac{1}{\sqrt{2}} e^{(2 \pi k / M)}, k=0,1, \ldots, M-1\right\} .
$$

Define the following matrices:

$$
\begin{aligned}
& \underline{A}_{0} \triangleq\left[\begin{array}{cc}
a_{0} & a_{1} \\
-a_{1}^{*} & a_{0}^{*}
\end{array}\right], \\
& \underline{A}_{n} \triangleq\left[\begin{array}{cc}
a_{2 n} & a_{2 n+1} \\
-a_{2 n+1}^{*} & a_{2 n}^{*}
\end{array}\right], \quad \text { for } n \geq 1, \\
& \underline{G}_{n} \triangleq \underline{A}_{n} \underline{A}_{0}^{H} .
\end{aligned}
$$

It is easy to see that $\underline{A}_{n}$ and $\underline{G}_{n}$ are both orthogonal matrices, that is, $\underline{A}_{n} \underline{A}_{n}^{H}=\underline{A}_{n}^{H} \underline{A}_{n}=\underline{G}_{n} \underline{G}_{n}^{H}=\underline{G}_{n}^{H} \underline{G}_{n}=\mathbf{I}_{2}$. Hence given $\underline{G}_{n}, \underline{A}_{n}$ can be obtained by

$$
\underline{A}_{n}=\underline{G}_{n} \underline{A}_{0} .
$$

The space-time differential block code is recursively defined as follows:

$$
\begin{gathered}
\underline{X}_{0}=\underline{A}_{0}, \\
\underline{X}_{n}=\underline{G}_{n} \underline{X}_{n-1}, \quad n=1,2, \ldots,
\end{gathered}
$$

By a simple induction, it is easy to show that the matrix $\underline{X}_{n}$ has the following form

$$
\underline{X}_{n} \triangleq\left[\begin{array}{cc}
x_{2 n} & x_{2 n+1} \\
-x_{2 n+1}^{*} & x_{2 n}^{*}
\end{array}\right],
$$

where $\left\|x_{2 n}\right\|^{2}+\left\|x_{2 n+1}\right\|^{2}=1$. Hence $\underline{X}_{n}$ is also an orthogonal matrix, and by (5), we have

$$
\underline{X}_{n} \underline{X}_{n-1}^{H}=\underline{G}_{n} .
$$

At time slot $2 n$, the symbols on the first row of $\underline{X}_{n}, x_{2 n}$, and $x_{2 n+1}$ are transmitted simultaneously from antenna 1 and antenna 2 , respectively. At time slot $2 n+1$, the symbols on the second row of $\underline{X}_{n},-x_{2 n+1}^{*}$, and $x_{2 n}^{*}$ are transmitted simultaneously from the two antennas. We first consider the case where the channel is static. Let $\alpha_{1}$ and $\alpha_{2}$ be the complex fading gains between the two transmit antennas and the receive antenna, respectively. The received signals at time slots $2 n$ and $2 n+1$ are then given, respectively, by

$$
\begin{aligned}
y_{2 n} & =\alpha_{1} x_{2 n}+\alpha_{2} x_{2 n+1}+v_{2 n}, \\
y_{2 n+1} & =-\alpha_{1} x_{2 n+1}^{*}+\alpha_{2} x_{2 n}^{*}+v_{2 n+1},
\end{aligned}
$$

where $v_{2 n}$ and $v_{2 n+1}$ are independent complex Gaussian noise samples. Note that from (8), in the absence of noise, we can write the following:

$$
\underbrace{\left[\begin{array}{cc}
y_{2 n}^{*} & y_{2 n+1}^{*} \\
y_{2 n+1} & -y_{2 n}
\end{array}\right]}_{\underline{Y}_{n}}=\underbrace{\left[\begin{array}{cc}
\alpha_{1}^{*} & \alpha_{2}^{*} \\
\alpha_{2} & -\alpha_{1}
\end{array}\right]}_{\underline{H}} \underbrace{\left[\begin{array}{cc}
x_{2 n}^{*} & -x_{2 n+1} \\
x_{2 n+1}^{*} & x_{2 n}
\end{array}\right]}_{\underline{X}_{n}^{H}} .
$$

Since

$$
\underline{H}^{H} \underline{H}=\left(\left|\alpha_{1}\right|^{2}+\left|\alpha_{2}\right|^{2}\right) \mathbf{I}_{2},
$$

then using (7) and (9), we have

$$
\begin{aligned}
\underline{Y}_{n}^{H} \underline{Y}_{n-1} & =\left(\left|\alpha_{1}\right|^{2}+\left|\alpha_{2}\right|^{2}\right) \underline{X}_{n} \underline{X}_{n-1}^{H} \\
& =\left(\left|\alpha_{1}\right|^{2}+\left|\alpha_{2}\right|^{2}\right) \underline{G}_{n} .
\end{aligned}
$$

Based on the above discussion, we arrive at the following differential space-time decoding algorithm.

Algorithm 1 (differential space-time decoding). Given the initial information symbol matrix $\underline{A}_{0}$, let $\underline{\hat{A}}_{0}=\underline{A}_{0}$. Form $\underline{Y}_{0}$ according to (9) using $y_{0}$ and $y_{1}$. For $n=1,2, \ldots$,

- Form the matrix $\underline{Y}_{n}$ according to (9) using $y_{2 n}$ and $y_{2 n+1}$.

- Obtain an estimate $\underline{\underline{G}}_{n}$ of $\underline{G}_{n}$ which is closest to $\underline{Y}_{n}^{H} \underline{Y}_{n-1}$.

- Perform the following mapping $\underline{\hat{A}}_{n}=\underline{\hat{G}}_{n} \underline{\hat{A}}_{0}$.

\section{MULTIPLE-SYMBOL DECISION-FEEDBACK STDC DECODING IN FADING CHANNELS}

We now consider decoding of the space-time differential block code in flat-fading channels. In such channels, the received signals become

$$
\begin{aligned}
y_{2 n} & =\alpha_{2 n}^{(1)} x_{2 n}+\alpha_{2 n}^{(2)} x_{2 n+1}+v_{2 n}, \\
y_{2 n+1} & =-\alpha_{2 n+1}^{(1)} x_{2 n+1}^{*}+\alpha_{2 n+1}^{(2)} x_{2 n}^{*}+v_{2 n+1},
\end{aligned}
$$

where $\left\{\alpha_{n}^{(1)}\right\}_{n}$ and $\left\{\alpha_{n}^{(2)}\right\}_{n}$ are the fading processes associated with the channels between the two transmit antennas and the receive antenna, which are modelled as mutually independent complex Gaussian variables with Jakes' correlation structure [19]. That is, each of them has normalized autocorrelation function

$$
R(n) \triangleq E\left\{\alpha_{m+n} \alpha_{m}^{*}\right\}=E_{s} J_{0}\left(2 \pi B_{d} n T\right),
$$


where $E_{s}$ is the average symbol energy, $J_{0}(\cdot)$ is the zerothorder Bessel function of the first kind, $T$ is the symbol interval, and $B_{d}$ is the maximum Doppler shift, which is proportional to the vehicle speed and carrier frequency. In order to simplify the receiver structure, we make the assumption that the channels remain constant over two consecutive symbol intervals, that is, $\alpha_{2 n}^{(1)}=\alpha_{2 n+1}^{(1)}$ and $\alpha_{2 n}^{(2)}=\alpha_{2 n+1}^{(2)}$. Then (12) can be written as

$$
\underbrace{\left[\begin{array}{c}
y_{2 n} \\
y_{2 n+1}
\end{array}\right]}_{\underline{y}_{n}}=\underbrace{\left[\begin{array}{cc}
x_{2 n} & x_{2 n+1} \\
-x_{2 n+1}^{*} & x_{2 n}^{*}
\end{array}\right]}_{\underline{X}_{n}} \underbrace{\left[\begin{array}{c}
\alpha_{2 n}^{(1)} \\
\alpha_{2 n}^{(2)}
\end{array}\right]}_{\underline{\alpha}_{n}}+\underbrace{\left[\begin{array}{c}
v_{2 n} \\
v_{2 n+1}
\end{array}\right]}_{\underline{\underline{v}}_{n}} .
$$

Denote

$$
\begin{aligned}
\mathbf{y}_{n} & =\left[\begin{array}{llll}
\underline{y}_{n}^{T} & \underline{y}_{n-1}^{T} & \cdots & \underline{y}_{n-N+1}^{T}
\end{array}\right]^{T}, \\
\alpha_{n} & =\left[\begin{array}{llll}
\underline{\alpha}_{n}^{T} & \underline{\alpha}_{n-1}^{T} & \cdots & \underline{\alpha}_{n-N+1}^{T}
\end{array}\right]^{T}, \\
\mathbf{v}_{n} & =\left[\begin{array}{llll}
\underline{v}_{n}^{T} & \underline{v}_{n-1}^{T} & \cdots & \underline{v}_{n-N+1}^{T}
\end{array}\right]^{T}, \\
\mathbf{X}_{n} & =\operatorname{diag}\left\{\underline{X}_{n}, \underline{X}_{n-1}, \ldots, \underline{X}_{n-N+1}\right\}, \\
\mathbf{G}_{n} & =\left[\begin{array}{llll}
\underline{G}_{n} & \underline{G}_{n-1} & \cdots & \underline{G}_{n-N+2}
\end{array}\right] .
\end{aligned}
$$

Then we have

$$
\mathbf{y}_{n}=\mathbf{X}_{n} \alpha_{n}+\mathbf{v}_{n}
$$

The conditional log-likelihood function is given by

$$
\log p\left(\mathbf{y}_{n} \mid \mathbf{G}_{n}\right)=-\mathbf{y}_{n}^{H} \mathbf{Q}_{G}^{-1} \mathbf{y}_{n}-\log \operatorname{det}\left(\mathbf{Q}_{G}\right)-2 N \log \pi,
$$

where

$$
\begin{aligned}
\mathbf{Q}_{G} & \triangleq E\left\{\mathbf{y}_{n} \mathbf{y}_{n}^{H}\right\} \\
& =\mathbf{X}_{n} E\left\{\alpha_{n} \alpha_{n}^{H}\right\} \mathbf{X}_{n}^{H}+\sigma^{2} \mathbf{I}_{2 N} \\
& =E_{S} \mathbf{X}_{n}\left(\Sigma_{N} \otimes \mathbf{I}_{2}\right) \mathbf{X}_{n}^{H}+\sigma^{2} \mathbf{I}_{2 N},
\end{aligned}
$$

where $\otimes$ denotes the Kronecker matrix product, and the normalized $N \times N$ autocorrelation matrix has elements given by (assume that the fading remains constant over two consecutive symbol intervals)

$$
\Sigma_{N}[i, j]=J_{0}\left(4 \pi B_{d} T(i-j)\right) .
$$

Note that $\mathbf{X}_{n} \mathbf{X}_{n}^{H}=\mathbf{X}_{n}^{H} \mathbf{X}_{n}=\mathbf{I}_{2 N}$, hence we have

$$
\begin{aligned}
\mathbf{Q}_{G}^{-1} & =E_{s}^{-1} \mathbf{X}_{n}\left[\left(\Sigma_{N}+\frac{\sigma^{2}}{E_{s}} \mathbf{I}_{N}\right) \otimes \mathbf{I}_{2}\right]^{-1} \mathbf{X}_{n}^{H}, \\
\operatorname{det}\left(\mathbf{Q}_{G}\right) & =\operatorname{det}\left(E_{s} \Sigma_{N} \otimes \mathbf{I}_{2}+\sigma^{2} \mathbf{I}_{2 N}\right) .
\end{aligned}
$$

Denote $\mathbf{T} \triangleq\left(\Sigma_{N}+\left(\sigma^{2} / E_{s}\right) \mathbf{I}_{N}\right)^{-1}$, then we have

$$
\left[\left(\Sigma_{N}+\frac{\sigma^{2}}{E_{s}} \mathbf{I}_{N}\right) \otimes \mathbf{I}_{2}\right]^{-1}=\mathbf{T} \otimes \mathbf{I}_{2} .
$$

The maximum likelihood decoding metric becomes

$$
\hat{\mathbf{G}}_{n}=\arg \min _{\mathbf{G}_{n}} \rho\left(\mathbf{G}_{n}\right) \triangleq \mathbf{y}_{n}^{H} \mathbf{X}_{n}\left(\mathbf{T} \otimes \mathbf{I}_{2}\right) \mathbf{X}_{n}^{H} \mathbf{y}_{n} .
$$

Note that since $\mathbf{T}=\left[t_{i j}\right]$ is symmetric, the above cost function $\rho\left(\mathbf{G}_{n}\right)$ can be written as

$$
\begin{aligned}
\rho\left(\mathbf{G}_{n}\right)= & \sum_{i=0}^{N-1} \sum_{j=0}^{N-1} t_{i, j} \underline{y}_{n-i}^{H} \underline{X}_{n-i} \underline{X}_{n-j}^{H} \underline{y}_{n-j} \\
= & \sum_{i=0}^{N-1} t_{i, i} \underline{y}_{n-i}^{H} \underline{X}_{n-i} \underline{X}_{n-i}^{H} \underline{y}_{n-i} \\
& +\sum_{i=0}^{N-1} \sum_{j \neq i} t_{i, j} \underline{y}_{n-i}^{H} \underline{X}_{n-i} \underline{X}_{n-j}^{H} \underline{y}_{n-j} \\
= & \sum_{i=0}^{N-1} t_{i, i}\left\|\underline{y}_{n-i}\right\|^{2} \\
& +2 \mathfrak{R}\left\{\sum_{i=0}^{N-1} \sum_{j=i+1}^{N-1} t_{i, j} \underline{y}_{n-i}^{H}\left(\prod_{l=i}^{j-1} \underline{G}_{n-l}\right) \underline{y}_{n-j}\right\},
\end{aligned}
$$

where (23) follows from (5). Since the first term in (23) is independent of $\mathbf{G}_{n}$, the decision rule (22) becomes

$$
\hat{\mathbf{G}}_{n}=\arg \min _{\mathbf{G}_{n}} \mathfrak{R}\left\{\sum_{i=0}^{N-1} \sum_{j=i+1}^{N-1} t_{i, j} \underline{y}_{n-i}^{H}\left(\prod_{l=i}^{j-1} \underline{G}_{n-l}\right) \underline{y}_{n-j}\right\} .
$$

Hence for detecting $\mathbf{G}_{n}$, it is necessary to calculate $M^{2(N-1)}$ metrics, where $M$ is the symbol constellation size. This corresponds to $M^{2(N-1)} /(N-1)$ metric calculations per code word decision, that is, the computational complexity grows exponentially with $N$. Although these metric calculations can be considerably simplified by using an algorithm developed in [12], a simpler and more efficient way to reduce complexity is to replace the previous symbol matrices $\underline{G}_{n-1}, \ldots, \underline{G}_{n-N+2}$ in (24) by decision-feedback matrices $\underline{\hat{G}}_{n-1}, \ldots, \underline{\hat{G}}_{n-N+2}$. In doing so, we obtain codeword-by-codeword decision instead of block decision. By omitting all summands which depend exclusively on decision-feedback symbols and thus do not influence the decision, (24) can be transformed into the following decision-feedback decoding rule for $\underline{G}_{n}$ :

$$
\hat{\underline{G}}_{n}=\arg \min _{\underline{G}_{n}} \Re\left\{\underline{y}_{n}^{H} \underline{G}_{n} \sum_{j=1}^{N-1} t_{0, j}\left(\prod_{l=1}^{j-1} \hat{\underline{G}}_{n-l}\right) \underline{y}_{n-j}\right\} .
$$

Finally, the decision-feedback space-time differential decoding algorithm is summarized as follows.

Algorithm 2 (multiple-symbol decision-feedback space-time differential decoding). Given the initial information symbol matrix $\underline{A}_{0}$, let $\underline{\hat{A}}_{0}=\underline{A}_{0}$.

- Compute the feedback metric coefficients from $\mathbf{T}=$ $\left(\Sigma_{N}+\left(\sigma^{2} / E_{s}\right) \mathbf{I}_{N}\right)^{-1}$, based on the decision memory order $N$, the fading statistic $\Sigma_{n}$, and the signal-to-noise ratio $E_{s} / \sigma^{2}$. 


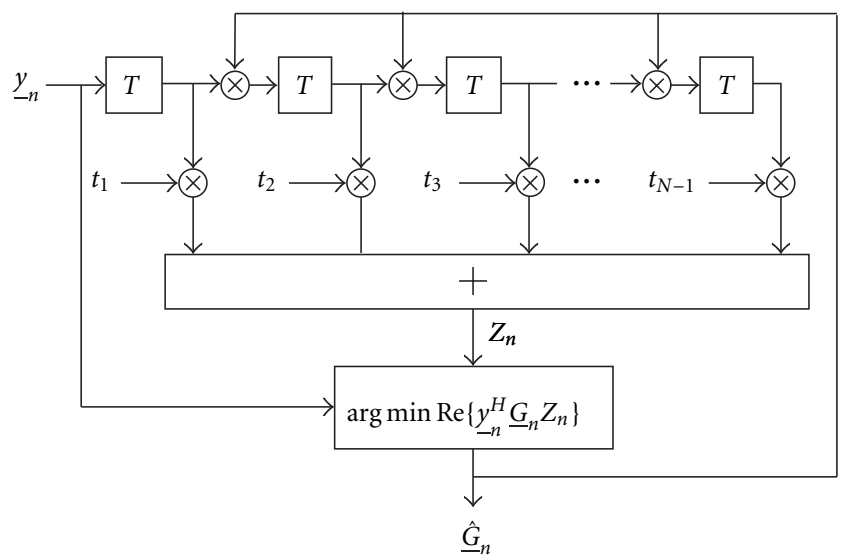

FIgURE 1: Structure of a decision-feedback space-time differential decoder.

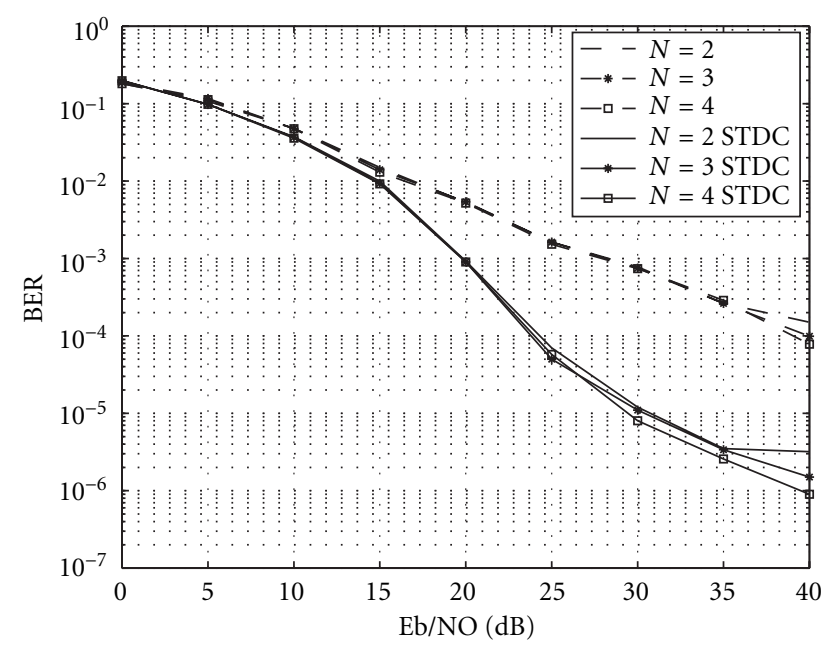

FIGURE 2: BER performance of decision-feedback space-time differential decoding in flat-fading channels with normalized Doppler $B_{d} T=0.003$. (Channels vary every two symbols).

- Estimate the initial symbol matrices: for $n=1,2, \ldots$, $N-1$,

- Estimate $\underline{\hat{G}}_{n}$ by simply quantizing $\underline{Y}_{n}^{H} \underline{Y}_{n-1}$.

- Perform the following mapping $\underline{\hat{A}}_{n}=\underline{\hat{G}}_{n} \underline{\hat{A}}_{0}$.

- For $n=N, N+1, \ldots$,

- Estimate $\hat{\underline{G}}_{n}$ according to (25).

- Perform the following mapping $\underline{\hat{A}}_{n}=\underline{\hat{G}}_{n} \underline{\hat{A}}_{0}$.

The structure of a decision-feedback space-time differential decoder is shown in Figure 1 , where $t_{j}=t_{0, j}$ for $j=$ $0,1, \ldots, N-1$.

\subsection{Simulation examples}

We consider a system with two transmit antennas and one receive antenna. QPSK constellation is employed in all simulations. By assuming that the fading processes remain constant over the duration of two symbol intervals, Figures 2, 3, and 4 show the BER performance of the decision-feedback

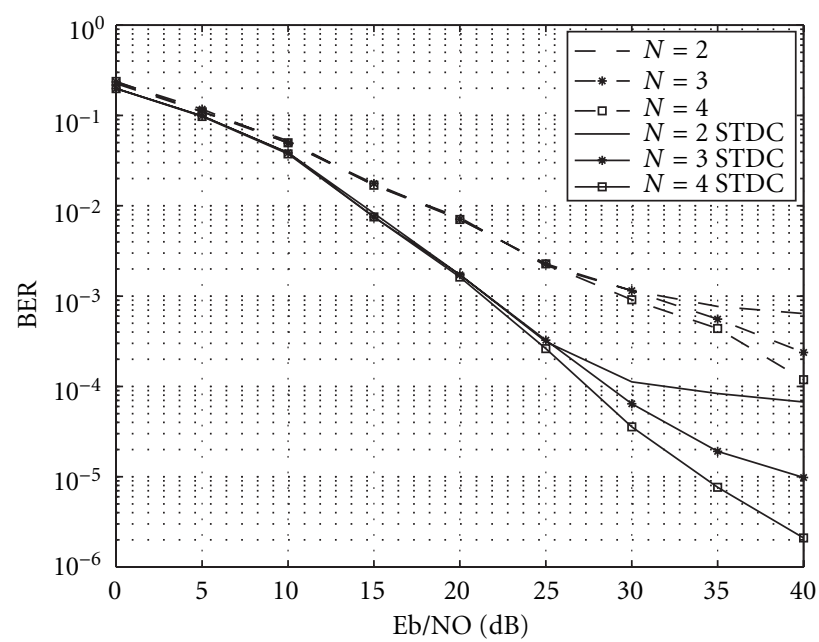

FIGURE 3: BER performance of decision-feedback space-time differential decoding in flat-fading channels with normalized Doppler $B_{d} T=0.0075$. (Channels vary every two symbols).

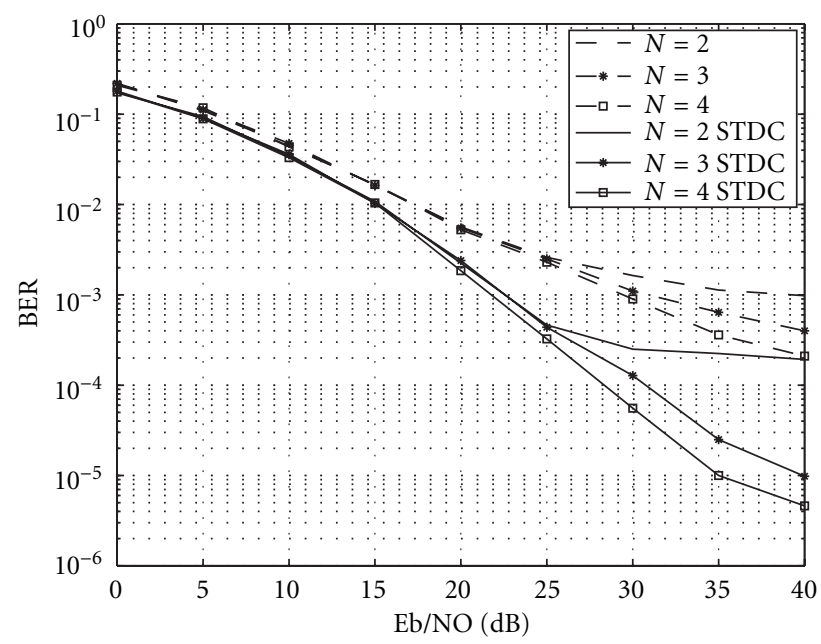

FIGURE 4: BER performance of decision-feedback space-time differential decoding in flat-fading channels with normalized Doppler $B_{d} T=0.01$. (Channels vary every two symbols).

space-time differential decoder in flat-fading channels with normalized Doppler $B_{d} T=0.003,0.0075$, and 0.01 , respectively. The performance of the single-antenna system is also shown. It is seen that space-time coding provides diversity gains over single-antenna systems. Moreover, the multiplesymbol decision-feedback decoding scheme reduces the error floor exhibited by the simple space-time differential decoding method in fading channels. Although the above multiple-symbol decoding scheme is derived based on the assumption that the fading remains constant over two consecutive symbols, little performance degradation is incurred when the channels actually vary from symbol to symbol. This is illustrated in Figures 5, 6, and 7, where the simulation conditions are the same as before except that the fading processes now vary from symbol to symbol. It is seen that the 


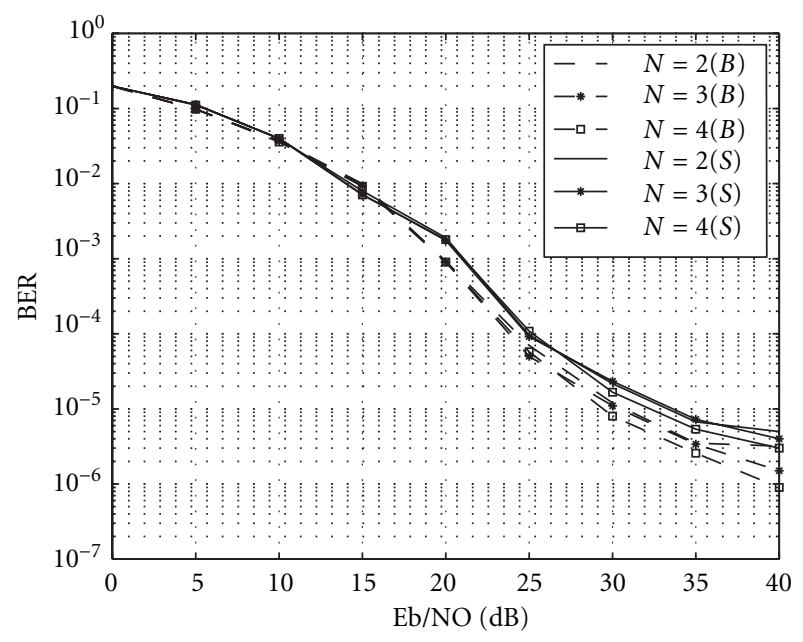

$B$-Channel changes block by block $S$-Channel changes symbol by symbol

FIGURE 5: BER performance of decision-feedback space-time differential decoding in flat-fading channels with normalized Doppler $B_{d} T=0.003$. (Channels vary every symbol).

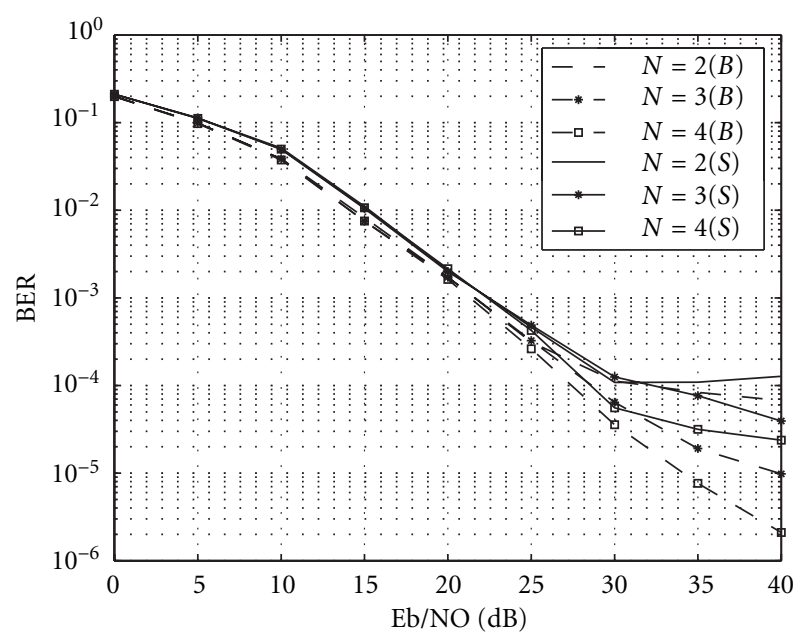

$B$-Channel changes block by block

$S$-Channel changes symbol by symbol

FIGURE 6: BER performance of decision-feedback space-time differential decoding in flat-fading channels with normalized Doppler $B_{d} T=0.0075$. (Channels vary every symbol).

performance degradation due to such a modelling mismatch is negligible for practical Doppler frequencies.

\section{APPLICATION TO OFDM SYSTEMS WITH SPACE-TIME CODING}

We next briefly discuss the application of decision-feedback space-time differential decoding in OFDM systems. Figure 8 shows the STDC-OFDM system structure. We consider a STDC-OFDM system with $Q$ subcarriers, two transmit antennas and one receive antenna, signaling through a

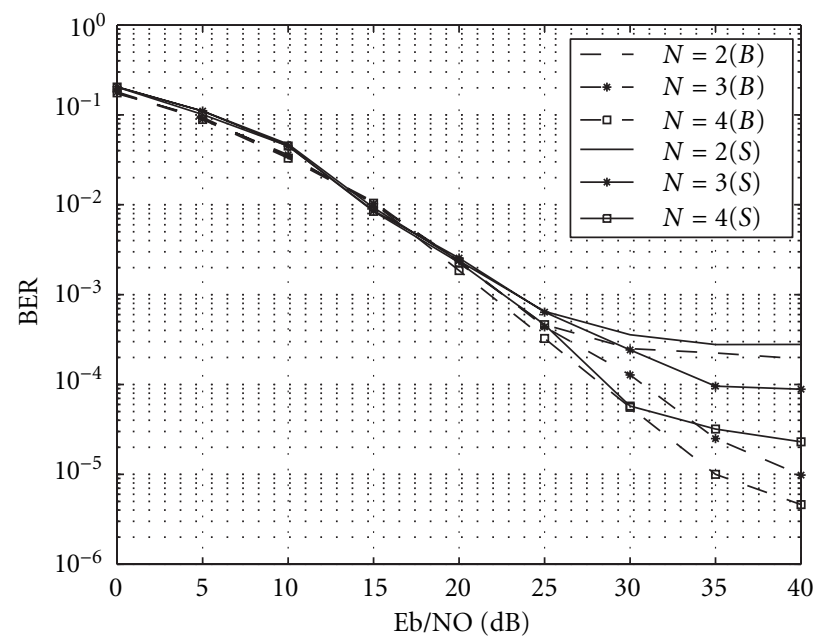

$B$-Channel changes block by block

$S$-Channel changes symbol by symbol

FIGURE 7: BER performance of decision-feedback space-time differential decoding in flat-fading channels with normalized Doppler $B_{d} T=0.01$. (Channels vary every symbol).

frequency- and time-selective fading channel. As illustrated in Figure 8, the information bits first go through a serial to parallel converter, then are encoded by a STDC encoder using the encoding algorithm introduced in Section 2. For each subcarrier $m, 0 \leq m<Q$, let $\left\{X_{m}[n]\right\}_{n}$ be the output of a space-time differential encoder. Then $\left\{X_{m}[n]\right\}_{m=0}^{Q-1}$ constitutes the OFDM symbol at time $n$. This OFDM symbol goes through an IDFT block to perform the inverse Fourier transform. Denote

$$
\begin{aligned}
\left\{x_{m}[2 n]\right\}_{m=0}^{Q-1} & =\operatorname{IDFT}\left[\left\{X_{m}[2 n]\right\}_{m=0}^{Q-1}\right] \\
\left\{x_{m}[2 n+1]\right\}_{m=0}^{Q-1} & =\operatorname{IDFT}\left[\left\{X_{m}[2 n+1]\right\}_{m=0}^{Q-1}\right] .
\end{aligned}
$$

After adding proper cyclic prefix, during time slot $2 n$, the signals $\left\{x_{m}[2 n]\right\}_{m=0}^{Q-1}$ are transmitted through antenna 1 serially, and $\left\{x_{m}[2 n+1]\right\}_{m=0}^{Q-1}$ are transmitted through antenna 2 serially; during time slot $2 n+1$, the signals $\left\{-x_{m}[2 n+1]^{*}\right\}_{m=0}^{Q-1}$ and $\left\{x_{m}[2 n]^{*}\right\}_{m=0}^{Q-1}$ are transmitted serially through antenna 1 and antenna 2 , respectively.

Now consider the channel response between the $i$ th transmit antenna and the receive antenna. Following [20], the time-domain channel impulse response can be modelled as a tapped-delay line, given by

$$
h^{(i)}(\tau ; n)=\sum_{l=0}^{L-1} \alpha_{l}^{(i)}[n] \delta(\tau-l T), \quad i=1,2,
$$

where $\delta(\cdot)$ is the Kronecker delta function; $L=\left\lceil\tau_{m} \Delta_{f}+1\right\rceil$ denotes the maximum number of resolvable taps, with $\tau_{m}$ being the maximum multipath spread and $\Delta_{f}$ being the tone spacing of the OFDM systems; $\alpha_{l}^{(i)}[n]$ is the complex amplitude of the $l$ th tap, whose delay is $l T$. Each of them is independent with each other and has the same normalized 


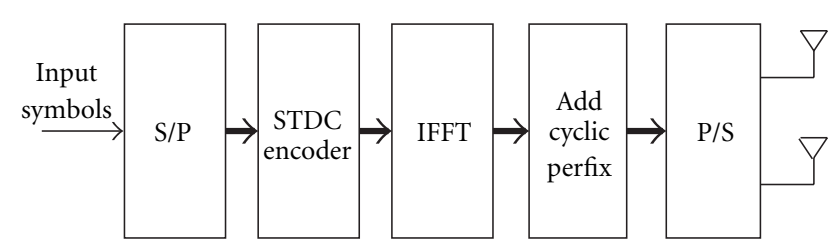

(a) Transmitter.

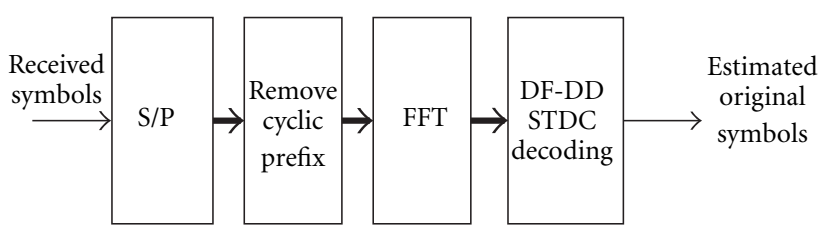

(b) Receiver.

FIGURE 8: STDC-OFDM system structure.

correlation function and different average powers $\sigma_{l}^{2}, l=$ $0,1, \ldots, L-1$. Assume Jakes' fading channel model, $R_{\alpha_{l}^{(i)}}(n)=$ $\sigma_{l}^{2} J_{0}\left(2 \pi B_{d} n T\right)$. For OFDM systems with proper cyclic extension and sample timing, with tolerable leakage, the channel frequency response between the $i$ th transmit antenna and the receive antenna at the $n$th time slot and at the $m$ th subcarrier can be expressed as

$$
\begin{aligned}
H_{m}^{(i)}[n]=\sum_{l=0}^{L-1} \alpha_{l}^{(i)}[n] e^{-j 2 \pi m l / Q}, \\
\quad i=1,2 ; m=0,1, \ldots, Q-1 .
\end{aligned}
$$

At the receiver, the received symbols first go through the serial to parallel converter. The received time-domain signals during two consecutive time slots are given, respectively, by

$$
\begin{aligned}
y_{m}[2 n]= & \sum_{l=0}^{L-1} \alpha_{l}^{(1)}[2 n] x_{m-l}[2 n] \\
& +\sum_{l=0}^{L-1} \alpha_{l}^{(2)}[2 n] x_{m-l}[2 n+1]+v_{m}[2 n], \\
y_{m}[2 n+1]= & -\sum_{l=0}^{L-1} \alpha_{l}[2 n+1] x_{m-l}[2 n+1]^{*} \\
& +\sum_{l=0}^{L-1} \alpha_{l}^{(2)}[2 n+1] x_{m-l}[2 n]^{*} \\
& +v_{m}[2 n+1], \quad m=0,1, \ldots, Q-1 .
\end{aligned}
$$

Assuming proper cyclic prefix is employed at each OFDM symbol. Then by applying DFT to the time-domain received signals (29), we obtain the frequency-domain received signals

$$
\begin{aligned}
Y_{m}[2 n]= & X_{m}[2 n] H_{m}^{(1)}[2 n] \\
& +X_{m}[2 n+1] H_{m}^{(2)}[2 n]+V_{m}[2 n], \\
Y_{m}[2 n+1]= & -X_{m}[2 n+1]^{*} H_{m}^{(1)}[2 n+1] \\
& +X_{m}[2 n]^{*} H_{m}^{(2)}[2 n+1]+V_{m}[2 n+1],
\end{aligned}
$$

where

$$
\begin{aligned}
\left\{Y_{m}[2 n]\right\}_{m=0}^{Q-1} & =\operatorname{DFT}\left[\left\{y_{m}[2 n]\right\}_{m=0}^{Q-1}\right], \\
\left\{Y_{m}[2 n+1]\right\}_{m=0}^{Q-1} & =\operatorname{DFT}\left[\left\{y_{m}[2 n+1]\right\}_{m=0}^{Q-1}\right],
\end{aligned}
$$

and where $H_{m}^{(1)}[n]$ and $H_{m}^{(2)}[n]$ are the complex channel frequency responses between the two transmit antennas and the receive antenna at time slot $n$ and the $m$ th subcarrier; $V_{m}[2 n]$ is the complex white Gaussian noise.

In order to use the multiple-symbol decision-feedback space-time differential decoding method, we need to analyze the auto-correlation of the channel frequency responses. Since the channel frequency responses are the DFT of channel impulse response, they are also complex Gaussian random variables. From (28), the auto-correlation function of $H_{m}^{(i)}$ is

$$
\begin{aligned}
R_{H_{m}^{(i)}}(n)= & E\left\{H_{m}^{(i)}[(n+j) T] H_{m}^{(i)}[j T]^{*}\right\} \\
= & E\left\{\sum_{l=0}^{L-1} \alpha_{l}^{(i)}[(n+j) T] e^{-j 2 \pi m l / Q}\right. \\
& \left.\cdot \sum_{q=0}^{L-1} \alpha_{q}^{(i)}[j T]^{*} e^{j 2 \pi m q / Q}\right\} \\
= & E\left\{\sum_{l=0}^{L-1} \alpha_{l}^{(i)}[(n+j) T] \alpha_{l}^{(i)}[j T]^{*}\right\} \\
= & \sum_{l=0}^{L-1} E\left\{\alpha_{l}^{(i)}[(n+j) T] \alpha_{l}^{(i)}[j T]^{*}\right\} \\
= & \sum_{l=0}^{L-1} R_{\alpha_{l}^{(i)}}(n) \\
= & J_{0}\left(2 \pi B_{d} n T\right) \cdot \sum_{l=0}^{L-1} \sigma_{l}^{2}, \quad m=0,1, \ldots, Q-1 .
\end{aligned}
$$

It is seen from (32) that the channel frequency responses have the same normalized autocorrelation function as the time-domain impulse responses. Hence, by assuming the channels remain static during two OFDM symbols, that is, $H_{m}^{(1)}[2 n]=H_{m}^{(1)}[2 n+1]$ and $H_{m}^{(2)}[2 n]=H_{m}^{(2)}[2 n+1]$, the multiple-symbol decision-feedback space-time differential decoding method discussed in Section 3 can be applied in parallel at each subcarrier $m$ to decode the information symbols.

Note that the cross-correlation of the channel frequency 


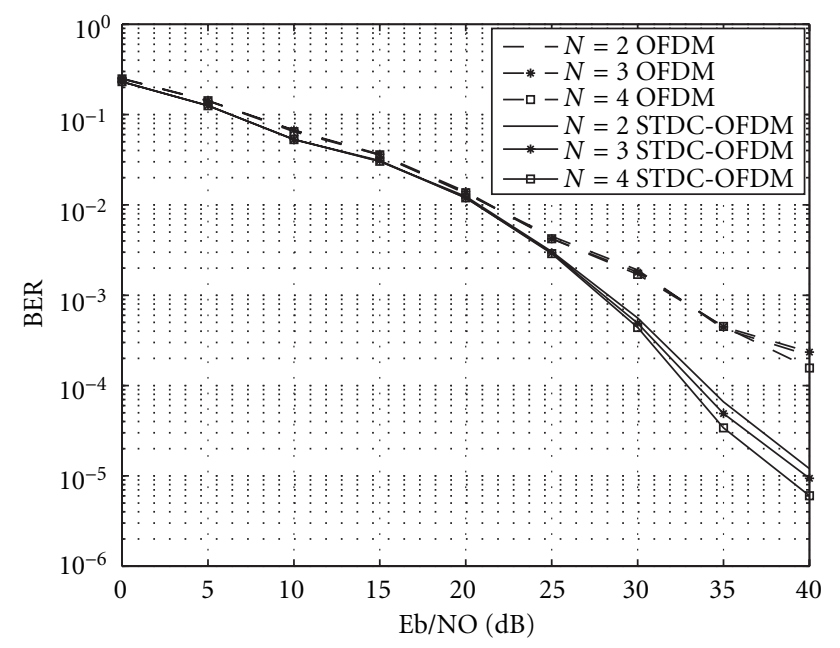

FIGURE 9: BER performance of decision-feedback space-time differential decoding in an OFDM system with $B_{d} T=0.003$. (Channels vary every OFDM symbol).

response is given by

$$
\begin{aligned}
R_{H_{k}^{(i)} H_{p}^{(i)}(n)=} & E\left\{H_{k}^{(i)}[(n+j) T] H_{p}^{(i)}[j T]^{*}\right\} \\
= & E\left\{\sum_{l=0}^{L-1} \alpha_{l}^{(i)}[(n+j) T] e^{-j 2 \pi k l / Q}\right. \\
& \left.\cdot \sum_{q=0}^{L-1} \alpha_{q}^{(i)}[j T]^{*} e^{j 2 \pi p q / Q}\right\} \\
= & E\left\{\sum_{l=0}^{L-1} \alpha_{l}^{(i)}[(n+j) T] \alpha_{l}^{(i)}[j T]^{*} e^{-j 2 \pi l(k-p) / Q}\right\} \\
= & \sum_{l=0}^{L-1} E\left\{\alpha_{l}^{(i)}[(n+j) T] \alpha_{l}^{(i)}[j T]^{*} e^{-j 2 \pi l(k-p) / Q}\right\} \\
= & \sum_{l=0}^{L-1} R_{\alpha_{l}^{(i)}}(n) e^{-j 2 \pi l(k-p) / Q} \\
= & J_{0}\left(2 \pi B_{d} n T\right) \cdot \sum_{l=0}^{L-1} \sigma_{l}^{2} \cdot e^{-j 2 \pi l(k-p) / Q}, \\
& k, p=0,1, \ldots, Q-1 .
\end{aligned}
$$

It is seen from (33) that the channel frequency responses at different carriers are no longer independent. Nevertheless for simplicity, the decision-feedback multiple-symbol STDC receiver decodes the symbols independently at each carrier, thereby ignoring the channel correlations.

\subsection{Simulation examples}

We consider a system with two transmit antennas and one receive antenna. The number of subcarriers in the OFDM system is $Q=128$. A 3-tap frequency selective channel with equal power between each transmit antenna and the receive antenna is assumed. The other parameters are the

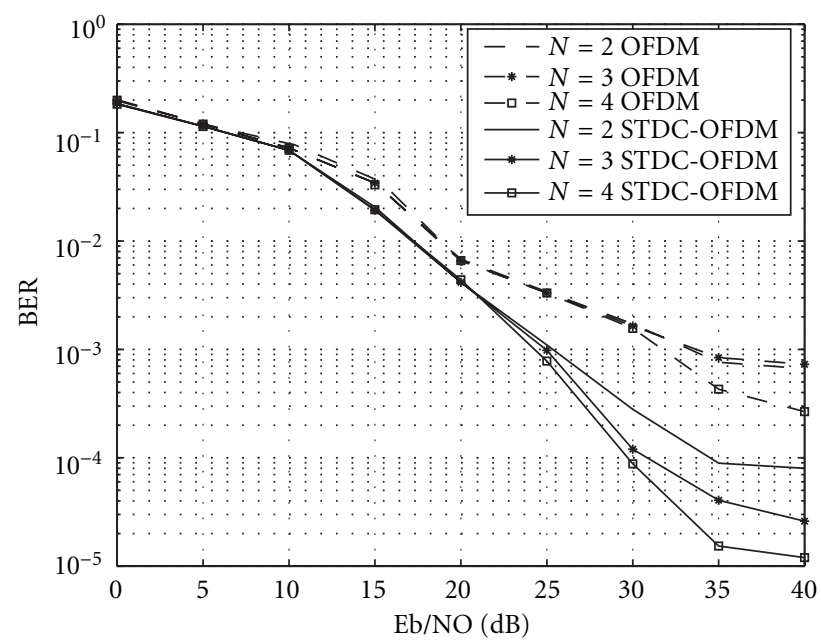

FIGURE 10: BER performance of decision-feedback space-time differential decoding in an OFDM system with $B_{d} T=0.0075$. (Channels vary every OFDM symbol).

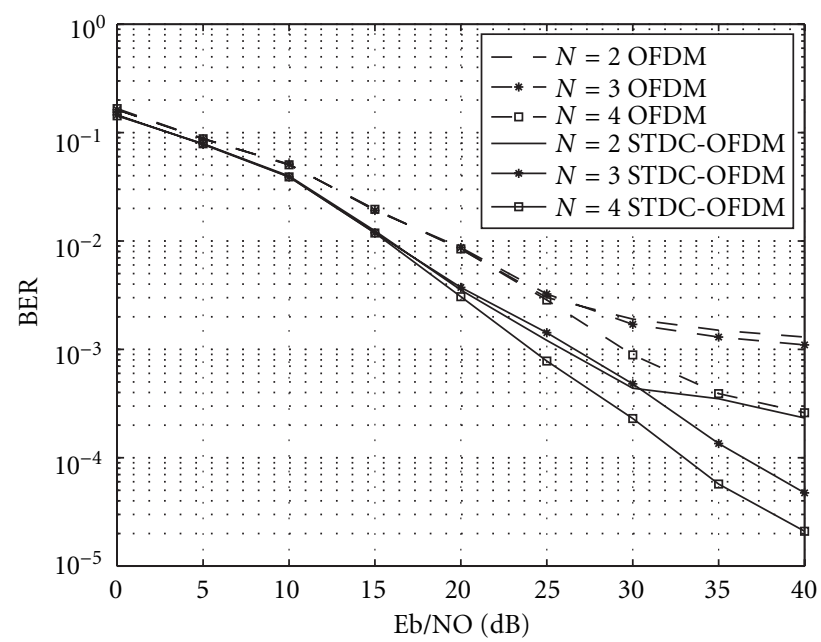

FIGURE 11: BER performance of decision-feedback space-time differential decoding in an OFDM system with $B_{d} T=0.01$. (Channels vary every OFDM symbol).

same as before. In the simulations, the fading processes vary from one OFDM symbol to another. Figures 9, 10, and 11 show the BER performance of the decision-feedback space-time decoding method in OFDM systems with different Doppler frequencies. Again it is seen that the decisionfeedback multiple-symbol STDC receiver reduces the error floor significantly in fast fading channels.

\section{CONCLUSION}

In this paper, we have developed a multiple-symbol decisionfeedback decoding algorithm for space-time differential code (STDC) in fading channels. This algorithm makes use of only the second-order statistic of the fading channels and has a very low computational complexity. It can considerably 
reduce the error floor of the conventional STDC decoding method, especially in fast fading channels. Although the algorithm is developed under the assumption that the fading channels remain static over one STDC codeword, that is, two symbol intervals, the performance loss is negligible when the channels actually vary within a codeword. Finally, we have also discussed the application of the proposed multiple-symbol decision-feedback STDC decoding algorithm in OFDM systems with frequency-selective fading.

\section{ACKNOWLEDGMENTS}

This work was supported in part by the U.S. National Science Foundation under grant CCR-9875314. The authors were with the Department of Electrical Engineering, Texas A\&M University, College Station, TX 77843-3128.

\section{REFERENCES}

[1] S. M. Alamouti, "A simple transmit diversity technique for wireless communications," IEEE Journal on Select Areas in Communications, vol. 16, no. 8, pp. 1451-1458, 1998.

[2] B. M. Hochwald and T. L. Marzetta, "Unitary spacetime modulation for multiple-antenna communications in Rayleigh flat fading," IEEE Transactions on Information Theory, vol. 46, no. 2, pp. 543-564, 2000.

[3] V. Tarokh, H. Jafarkhani, and A. R. Calderbank, "Space-time block codes from orthogonal designs," IEEE Transactions on Information Theory, vol. 45, no. 5, pp. 1456-1467, 1999.

[4] V. Tarokh, H. Jafarkhani, and A. R. Calderbank, "Space-time block coding for wireless communications: performance results," IEEE Journal on Selected Areas in Communications, vol. 17, no. 3, pp. 451-460, 1999.

[5] V. Tarokh, H. Jafarkhani, and A. R. Calderbank, "Spacetime codes for high data rate wireless communication: performance criterion and code construction," IEEE Transactions on Information Theory, vol. 44, no. 2, pp. 744-765, 1998.

[6] B. Lu and X. Wang, "Iterative receivers for multiuser spacetime coding systems," IEEE Journal on Selected Areas in Communications, vol. 18, no. 11, pp. 2322-2335, 2000.

[7] B. L. Hughes, "Differential space-time modulation," in Proc. 1999 Wireless Communications and Networking Conference, pp. 543-564, New Orleans, La, USA, September 1999.

[8] V. Tarokh and H. Jafarkhani, "A differential detection scheme for transmit diversity," IEEE Journal on Selected Areas in Communications, vol. 18, no. 7, pp. 1169-1174, 2000.

[9] D. Divsalar and M. K. Simon, "Multiple-symbol differential detection of MPSK," IEEE Trans. Communications, vol. 38, no. 3, pp. 300-308, 1990.

[10] D. Divsalar and M. K. Simon, "Maximum-likelihood differential detection of uncoded and trellis coded amplitude phase modulation over AWGN and fading channels-metrics and performance," IEEE Trans. Communications, vol. 42, no. 1, pp. 76-89, 1994.

[11] P. Ho and D. Fung, "Error performance of multiple symbol differential detection of PSK signals transmitted over correlated Rayleigh fading channels," IEEE Trans. Communications, vol. 40, no. 7, pp. 1566-1569, 1992.

[12] K. M. Mackenthun, "A fast algorithm for multiple-symbol differential detection of MPSK," IEEE Trans. Communications, vol. 42, no. 2/3/4, pp. 1471-1474, 1994.

[13] R. Schober, W. H. Gerstacker, and J. B. Huber, "Decisionfeedback differential detection of MDPSK for flat Rayleigh fading channels," IEEE Trans. Communications, vol. 47, no. 7, pp. 1025-1035, 1999.

[14] L. J. Cimini, "Analysis and simulation of a digital mobile channel using orthogonal frequency division multiplexing," IEEE Trans. Communications, vol. 33, no. 7, pp. 665-675, 1985.

[15] I. Kalet, "The multitone channel," IEEE Trans. Communications, vol. 37, no. 2, pp. 119-124, 1989.

[16] Y. (G.) Li, L. J. Cimini, and N. R. Sollenberger, "Robust channel estimation for OFDM systems with rapid dispersive fading channels," IEEE Trans. Communications, vol. 46, no. 7, pp. 902-915, 1989.

[17] Y. (G.) Li, N. Seshadri, and S. Ariyavisitakul, "Channel estimation for OFDM systems with transmitter diversity in mobile wireless channels," IEEE Journal on Selected Areas in Communications, vol. 17, no. 3, pp. 461-471, 1999.

[18] B. Lu, X. Wang, and Y. (G.) Li, "Iterative receivers for spacetime block coded OFDM systems in dispersive fading channels," IEEE Transactions on Wireless Communications, vol. 1, no. 2, 2002.

[19] W. C. Jakes, Microwave Mobile Communications, WileyInterscience, New York, NY, USA, 1974.

[20] J. G. Proakis, Digital Communications, McGraw-Hill, New York, NY, USA, 2nd edition, 1989.

Yan Liu received her B.E. and M.E. degrees in Electrical Engineering in 1992 and 1995, respectively, from Dalian University of Technology, Dalian, China; the M.S. degree in Electrical Engineering from Texas A\& M University, College Station, TX, in 2001. From 1995 to 1998 , she was a lecturer with Dalian Maritime University, Dalian, China. In September 2001, she joined Schlumberger Technology Corporation, working in the Software Metier group.

Xiaodong Wang received the B.S. degree in Electrical Engineering and Applied Mathematics (with the highest honor) from Shanghai Jiao Tong University, Shanghai, China, in 1992; the M.S. degree in Electrical and Computer Engineering from Purdue University in 1995; and the Ph.D. degree in Electrical Engineering from Princeton University in 1998. From July 1998 to December 2001, he was an Assistant Professor in the Depart-

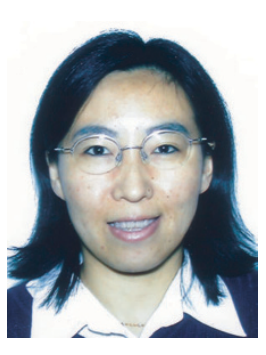
ment of Electrical Engineering, Texas A\&M University. In January 2002, he joined the Department of Electrical Engineering, Columbia University, as an Assistant Professor. Dr. Wang's research interests fall in the general areas of computing, signal processing and communications. He has worked in the areas of digital communications, digital signal processing, parallel and distributed computing, nanoelectronics and quantum computing, and has published extensively in these areas. His current research interests include multiuser communications theory and advanced signal processing for wireless communications. He worked at the AT\&T Labs-Research, in Red Bank, NJ, during the summer of 1997. Dr. Wang is a member of the IEEE and a member of the American Association for the Advancement of Science. He received the 1999 NSF CAREER Award, and the 2001 IEEE Communications Society and Information Theory Society Joint Paper Award. He currently serves as an Associate Editor for the IEEE Transactions on Communications, and for the IEEE Transactions on Signal Processing, and for the IEEE Transactions on Wireless Communications. 